\title{
Excitatory Synaptogenesis between Identified Lymnaea Neurons Requires Extrinsic Trophic Factors and Is Mediated by Receptor Tyrosine Kinases
}

\author{
Toshiro Hamakawa, ${ }^{1,2}$ Melanie A. Woodin, ${ }^{1}$ Micki C. Bjorgum, ${ }^{1}$ Sherry D. Painter, ${ }^{3}$ Mayumi Takasaki, ${ }^{2}$ \\ Ken Lukowiak, ${ }^{1}$ Gregg T. Nagle, ${ }^{3}$ and Naweed I. Syed ${ }^{1}$ \\ ${ }^{1}$ Respiratory and Neuroscience Research Groups, Faculty of Medicine, University of Calgary, Calgary, Alberta, Canada \\ T2N 4N1, 2Department of Anesthesiology, Miyazaki Medical College, Miyazaki 889-1692, Japan, and 3Marine Biomedical \\ Institute and Department of Anatomy and Neurosciences, University of Texas Medical Branch, Galveston, Texas 77555
}

Neurotrophic factors have well established roles in neuronal development and adult synaptic plasticity, but their precise role in synapse formation has yet to be determined. This paper provides the first direct evidence that neurotrophic factors in brain conditioned medium (CM) differentially regulate excitatory and inhibitory synapse formation. Somata of identified presynaptic and postsynaptic neurons were isolated from the CNS of Lymnaea and were cultured in a soma-soma configuration in the presence (CM) or absence [defined medium (DM)] of trophic factors. In DM, excitatory synapses did not form. When they were paired in CM or in DM containing Lymnaea epidermal growth factor (EGF); however, all presynaptic neurons reestablished their specific excitatory synapses, which had electrical properties similar to those seen in vivo. CM-induced formation of excitatory synapses required transcription and de novo pro- tein synthesis, as indicated by the observations that synapse formation was blocked by the protein synthesis inhibitor anisomycin and the protein transcription blocker actinomycin D; the CM factor was inactivated by boiling. They were also blocked by receptor tyrosine kinase inhibitors (lavendustin A, genistein, K252a, and KT5926) but not by inactive analogs (genistin and lavendustin B), suggesting that the effect was mediated by receptor tyrosine kinases. These results, together with our previously published data, demonstrate that trophic factors are required for excitatory, but not inhibitory, synapse formation and extends the role of EGF from cell proliferation, neurite outgrowth, and survival to excitatory synapse formation.

Key words: synapse formation; trophic factors; EGF; protein synthesis; trk receptors; Lymnaea; soma-soma synapses
Neurotrophic factors are well known for their involvement in neuronal survival, differentiation, and neurite outgrowth (see Purves and Lichtman, 1985; Oppenheim, 1991; Thoenen, 1991). In addition to these well defined developmental roles, they also have effects on the adult mammalian nervous system (for review, see Thoenen, 1995; Berninger and Poo, 1996; Schuman, 1997). Recent studies have shown that the neurotrophins alter the efficacy of synaptic transmission at a neuromuscular synapse in vitro (Lohof et al., 1993; Stoop and Poo, 1995, 1996; Wang et al., 1995; Liou et al., 1997; Wang and Poo, 1997) and modulate synaptic transmission between cortical and hippocampal neurons in culture (Kim et al., 1994; Levine et al., 1995; Leßmann et al., 1994; Li et al., 1998) and in hippocampal slices (Kang and Schuman, 1995). Recent studies have shown that various members of the neurotrophin family exert differential effects on excitatory and inhibitory synapses (Vicario-Abéjon et al., 1998): brain-derived neurotrophic factor (BDNF), for example, promotes both excitatory and inhibitory synapse formation, whereas neurotrophin-3 induces the formation of excitatory synapses only (Vicario-

\footnotetext{
Received May 11, 1999; revised Aug. 12, 1999; accepted Aug. 16, 1999.

This work was supported by the Medical Research Council (MRC) of Canada. N.I.S. is an Alberta Heritage Foundation for Medical Research (AHFMR) Senior Scholar; M.W. was supported by studentship awards from the AHFMR and MRC. Excellent technical support by Wali Zaidi is also acknowledged.

T.H. and M.A.W. contributed equally to this study.

Correspondence should be addressed to Dr. Naweed I Syed, Department of Anatomy, Faculty of Medicine. University of Calgary, Calgary, Alberta, Canada T2N 4N1. E-mail: nisyed@ucalgary.ca.

Copyright (C) 1999 Society for Neuroscience 0270-6474/99/199306-07\$05.00/0
}

Abéjon et al., 1998). They can also modulate synaptic activity; BDNF reduces both evoked and spontaneous IPSPs without appreciably affecting evoked EPSPs (Tanaka et al., 1997). Despite their well defined actions, relatively little is known about the cellular and molecular mechanisms by which neurotrophins effect synaptic plasticity in the nervous system.

This lack of knowledge is attributable in part to the complexity of the mammalian brain, in which specific synapse formation between defined sets of presynaptic and postsynaptic neurons can only rarely be studied directly. Moreover, because neurite outgrowth that precedes synapse formation relies completely on extrinsic growth factors; their involvement in synapse formation, independent of neurite outgrowth, cannot be studied directly.

To obtain synapses between identified presynaptic and postsynaptic neurons in the absence of neurite outgrowth, we recently developed a soma-soma synapse preparation using Lymnaea neuron right pedal dorsal 1 (RPeD1) and visceral dorsal 4 (VD4) (Feng et al., 1997). We demonstrated that, when juxtaposed in cell culture, appropriate inhibitory synapses develop between the somata of these identified neurons, which are morphologically and electrophysiologically similar to those seen in vivo. These synapses require de novo protein synthesis but not extrinsic growth factors or substrate adhesion molecules (Feng et al., 1997). We demonstrate in the present study, however, that excitatory synapse formation between a variety of presynaptic and postsynaptic neurons depends on extrinsic trophic factors. Moreover, trophic factor-induced excitatory synaptogenesis between identified Lymnaea neurons is independent of neurite outgrowth, 
requires new protein synthesis, and is mediated by receptor tyrosine kinases. Taken together, the data in this study are consistent with our hypothesis that inhibitory and excitatory synapse formation in the nervous system is differentially regulated by trophic factors. Furthermore, this study provides the first direct information about the mechanisms by which trophic factorinduced excitatory synapse formation is regulated between identified neurons.

\section{MATERIALS AND METHODS}

Animals. Stocks of the fresh water pond snail Lymnaea stagnalis were maintained at room temperature in an aquarium containing well aerated, filtered pond water and were fed lettuce. Snails $\sim 1-2$ months old (shell length, $10-15 \mathrm{~mm}$ ) were used for studies involving cell isolation; animals 2-3 months old (shell length, $15-25 \mathrm{~mm}$ ) were used to produce brain conditioned medium (CM).

Cell culture. Single cells were isolated and cultured as described previously (Syed et al., 1990; Ridgway et al., 1991). Briefly, snails were anesthetized with 10\% Listerine (ethanol, 21.9\%; menthol, $0.042 \%$ ) solution in normal saline (in $\mathrm{mm}$ ): $51.3 \mathrm{NaCl}, 1.7 \mathrm{KCl}, 4.0 \mathrm{CaCl}_{2}$, and 1.5 $\mathrm{MgCl}_{2}$ ) buffered to $\mathrm{pH} 7.9$ with HEPES. The central ring ganglia were removed under sterile conditions and washed in a series of antibiotic washes (gentamycin, $50 \mu \mathrm{g} / \mathrm{ml}$; three washes, $15 \mathrm{~min}$ each). The antibiotic-treated ganglia were then enzyme-treated and pinned down to the bottom of a dissection dish.

Identified cells (somata and initial axon stumps) were isolated by applying gentle suction through a fire-polished and Sigmacote (Sigma, St. Louis, MO)-treated pipette. Isolated cells were plated onto poly-L-lysinepretreated coverslips (Ridgway et al., 1991) in the presence of defined medium (DM; L-15; Life Technologies, Gaithersburg, MD; special order), CM, or DM containing Lymnaea epidermal growth factor (EGF) (Nagle et al., 1998). Isolated somata of identified neurons were juxtaposed in a soma-soma configuration as previously reported (Feng et al., 1997) and left undisturbed overnight.

To prepare $\mathrm{CM}$, gentamycin $(20 \mu \mathrm{g} / \mathrm{ml})$-treated ganglia were incubated in DM contained in Sigmacote-treated glass Petri dishes. DM consisted of serum-free $50 \% \mathrm{~L}-15$ medium with added inorganic salts (in mM: $40 \mathrm{NaCl}, 1.7 \mathrm{KCl}, 4.1 \mathrm{CaCl}_{2}, 1.5 \mathrm{MgCl}_{2}$, and $10 \mathrm{HEPES}, \mathrm{pH} 7.9$ ) and $20 \mu \mathrm{M}$ gentamycin. Ganglia were maintained in a humidified glass chamber for $3 \mathrm{~d}$ (for details, see Wong et al., 1981). The central ring ganglia were subsequently removed, and the $\mathrm{CM}$ was frozen $\left(-20^{\circ} \mathrm{C}\right)$ until it was used. Heat-inactivated $\mathrm{CM}$ was prepared by boiling $\mathrm{CM}$ for $20 \mathrm{~min}$.

Electrophysiology. Conventional intracellular recording techniques, as described previously (Syed and Winlow, 1991), were used to monitor neuronal activity. Glass microelectrodes (1.5-2.0 $\mu \mathrm{m}$ internal diameter; World Precision Instruments, Sarasota, FL) were filled with a saturated solution of $\mathrm{K}_{2} \mathrm{SO}_{4}$ (resistance, $20-40 \mathrm{M}[\mathrm{SCAP}] \Omega$ ). Neurons were viewed under an inverted microscope (Axiovert 135; Zeiss, Thornwood, NY) and impaled using Narashige (Tokyo, Japan) micromanipulators (MM 202 and MM 204). The electrical signals were amplified (Neuro Data Instrument Corp.) and displayed on a digital storage oscilloscope (PM 3394; Philips, Eindhoven, The Netherlands) and recorded on a chart recorder (TA 240S; Gould, Cleveland, OH).

Sulforhodamine was obtained from Molecular Probes (Eugene, OR; 6-359). All other chemicals were obtained from Sigma unless otherwise stated.

Dye injection. Soma-soma paired cells were labeled with either Lucifer yellow or sulforhodamine. Electrode tips were filled with dye [Lucifer yellow $(3 \%)$ or sulforhodamine $(10 \%)]$, and then the electrode was back-filled with $\mathrm{LiCl}(0.1 \%)$. Both dyes were injected iontophoretically as previously described (Syed and Winlow, 1989). The injected cells were fixed with $4 \%$ paraformaldehyde for 1-2 $\mathrm{hr}$ and viewed under a fluorescence microscope (Zeiss Axioskop). The labeled cells were imaged either by a cooled CCD camera (Photometrics, Tucson, AZ) or photographed with a $35 \mathrm{~mm}$ camera (Zeiss MC 80).

\section{RESULTS}

We have previously demonstrated that the inhibitory synapse between identified Lymnaea neurons RPeD1 and VD4 reforms in a soma-soma configuration in DM (Feng et al., 1997). These data led us to believe that synaptogenesis between inhibitory partners
A
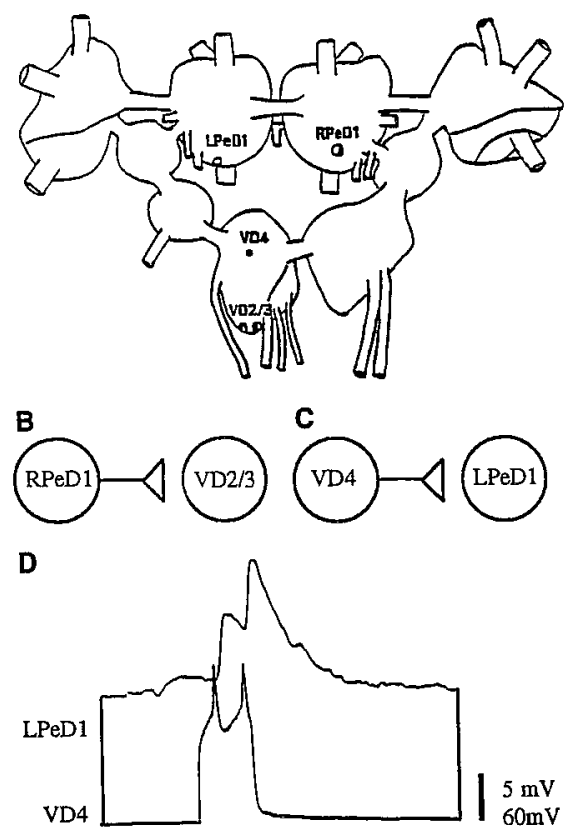

Figure 1. Diagrams indicating the position, location, and nature of synaptic connections between identified Lymnaea neurons used in the present study. $A$, Ganglionic location of identified neurons right pedal dorsal 1 (RPeD1), left pedal dorsal 1 ( $L P e D 1)$, visceral dorsal 4 (VD4), and visceral dorsal $2 / 3(V D 2 / 3)$, which are located in the right pedal, left pedal, and visceral ganglia, respectively. Note that VD2 and 3 are thought to be electrophysiologically and morphologically identical and are therefore simply referred to as VD2/3 (Magoski and Bulloch, 1997). B, Diagrammatic representation of the in vivo excitatory synaptic connection between RPeD1 and VD2/3 (Winlow and Benjamin, 1977; Magoski et al., 1995; Magoski and Bulloch, 1997). RPeD1 forms an excitatory synapse with VD2/3. C, Diagrammatic representation of the in vivo synaptic connection between VD4 and LPeD1. VD4 forms an excitatory synapse with LPeD1. $D$, Simultaneous intracellular recording in an isolated ganglionic preparation revealed an excitatory synapse between VD4 and LPeD1; action potentials in VD4 produced 1:1 EPSPs in LPeD1.

may rely exclusively on intrinsic cell-cell signaling mechanisms, independent of extrinsic growth factors (Feng et al., 1997). In the present study, we examined whether excitatory synapses could also reform in vitro in the absence of CM-derived trophic factors. We began with identified presynaptic and postsynaptic neurons whose in vivo synaptic connections are well characterized. Specifically, we examined the presynaptic cells RPeD1 and VD4 and their postsynaptic partners visceral dorsal $2 / 3$ (VD2/3) and left pedal dorsal 1 (LPeD1), respectively (Fig. $1 A$ ). In the intact ganglia, RPeD1 makes monosynaptic excitatory synapses with VD2/3 (Fig. 1B; Magoski et al., 1995), and VD4 makes an excitatory synapse with $\operatorname{LPeD} 1(n=13)$ (Fig. $1 C)$.

\section{Excitatory synapses between identified Lymnaea neurons fail to develop in defined medium}

To determine whether in vivo excitatory synapses reform in a soma-soma configuration in vitro, isolated somata were juxtaposed on poly-L-lysine dishes containing DM alone. After either 12-24 or 24-72 hr of soma-soma pairing, simultaneous intracellular recordings were made from both cells. Sharp electrode recordings routinely failed to reveal electrophysiologically detectable synaptic connections between soma-soma paired cells. Both single action potentials (data not shown) and bursts of action potentials in RPeD1 failed to induce EPSPs in VD2/3 $(n=64)$ 

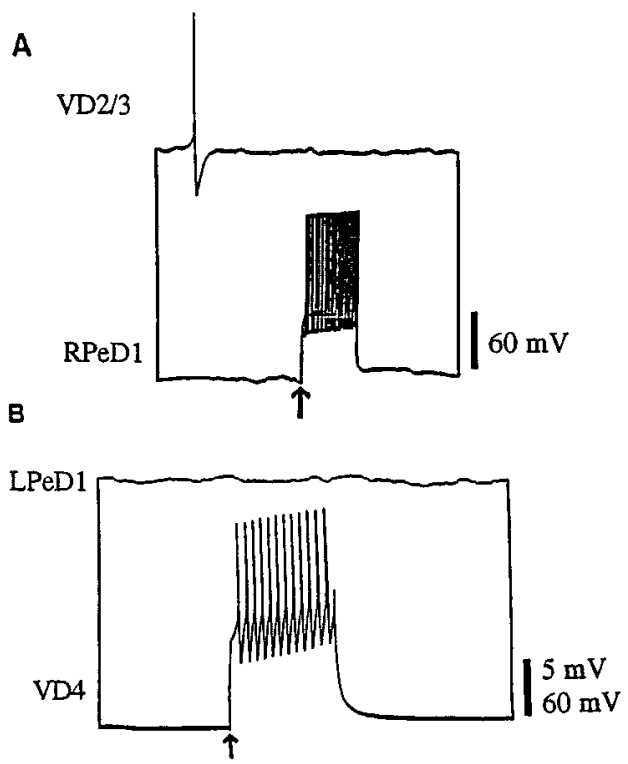

Figure 2. Excitatory synapses between soma-soma paired neurons fail to develop in DM. After 18-24 hr of cell pairing in DM, simultaneous intracellular recordings revealed that a burst of action potentials in $\mathrm{RPeD} 1$ failed to generate a postsynaptic potential in VD2/3 $(A)$. Likewise, a burst of action potentials in VD4 failed to alter the membrane potential of LPeD1 $(B)$.

(Fig. 2A). Similarly, a burst of action potentials in VD4 failed to generate an excitatory response in LPeD1 $(n=16)$ (Fig. 2B). These data showed that, unlike the inhibitory synapses (Feng et al., 1997), excitatory synapses between presynaptic and postsynaptic neurons fail to develop in DM.

To test the generality of these observations, we soma-soma paired a number of identified presynaptic and postsynaptic neurons in DM and tested for excitatory synapses electrophysiologically. We found that excitatory synapses did not develop between any of the cell pairs tested (Table 1). These data are consistent with our observations that neither cell-cell contact nor DM alone is sufficient to promote synapse formation between the excitatory partners.

\section{$\mathrm{CM}$ is necessary for synaptogenesis between excitatory partners}

To test whether soma-soma synapses between excitatory partners require growth factors, cells were paired in CM. Specifically, isolated somata of both presynaptic and postsynaptic cells were soma-soma paired on poly-L-lysine-treated dishes containing CM. After 12-18 hr, the presence of synaptic transmission was examined electrophysiologically by monitoring the intracellular activity of both cells. We found that action potentials in RPeD1 produced 1:1 EPSPs in VD2/3 (100\%; $n=24)$ (Fig. $3 A)$. These synapses had electrophysiological properties similar to those described in vivo (Winlow and Benjamin, 1977; Magoski et al., 1995; Magoski and Bulloch, 1997). Intracellular recordings from VD4 and LPeD1 revealed that an excitatory synapse had also formed between these cells. Single action potentials in the presynaptic cell reliably produced 1:1 EPSPs in the postsynaptic neuron $(n=$ 20) (see Fig. 3B, Table 1). This synapse was similar to that seen in vivo (Fig. 1).

To determine whether the CM factor(s) required for excitatory synapse formation are proteins, $\mathrm{CM}$ was pretreated by boiling for
$20 \mathrm{~min}$ before cell-cell pairing. After $18-24 \mathrm{hr}$ in heatinactivated CM, simultaneous intracellular recordings were made from RPeD1 and VD4; they showed that no excitatory synapses had formed (100\%; $n=10$ ) (Fig. 4). This suggests that the CM factor(s) mediating excitatory synapse formation are heatsensitive and are likely to be proteins.

To rule out the possibility that the $\mathrm{CM}$-induced excitatory synaptogenesis between RPeD1 and VD2/3 and between VD4 and LPeD1 was simply a cell type-specific phenomenon, excitatory synapses between other presynaptic and postsynaptic cells were also reconstructed in CM. We found that excitatory synapses between various pairs of presynaptic and postsynaptic cells also reformed in $\mathrm{CM}$, suggesting that the $\mathrm{CM}$ requirement for excitatory synapse formation extends to other cell pairs as well (Table 1).

To exclude the possibility that CM-induced excitatory synaptogenesis might be caused by trophic factor-induced neurite outgrowth on the substrate or the corresponding partner, cells were paired in CM, and their morphological profiles were examined after injection of presynaptic and postsynaptic neurons with different fluorescent dyes. When VD2/3 was injected with sulforhodamine and RPeD1 was injected with Lucifer yellow, neither presynaptic nor postsynaptic cell exhibited neurite outgrowth in $\mathrm{CM}(n=7)$ (Fig. 5). This illustrates that CM-induced excitatory synaptogenesis does not appear to involve neuronal sprouting.

These data collectively demonstrate that (1) CM is required for excitatory synapses to form; (2) the synapse-promoting CM factors are likely to be proteins; and (3) CM-induced excitatory synaptogenesis does not involve neurite outgrowth.

To examine the cellular mechanisms underlying CM-induced plasticity of synaptic connections between Lymnaea neurons, we focused our analysis on the RPeD1 and VD2/3 synapse. This pair was selected because RPeD1 uses dopamine as its sole neurotransmitter (McCaman et al., 1979; Magoski et al., 1995), ruling out the possibility that CM-induced excitatory synapse formation involved either (1) a switch in the neurotransmitter of the presynaptic cell or (2) the induction of an unidentified mediator responsible for excitatory synaptic transmission.

\section{CM-induced excitatory synaptogenesis between RPeD1 and VD2/3 requires de novo protein synthesis and transcription}

To test whether CM-induced excitatory synaptogenesis is contingent on de novo protein synthesis and transcription, cells were soma-soma paired in CM containing either a protein synthesis inhibitor (anisomycin; $12.5 \mu \mathrm{g} / \mathrm{ml}$ ) or a protein transcription blocker (actinomycin D; $1 \mu \mathrm{g} / \mathrm{ml}$ ). Simultaneous intracellular recordings from RPeD1 and VD2/3 after 12-24 hr of pairing did not reveal electrophysiologically detectable synapses between the cells. To rule out the possibility that the carrier solution might have perturbed synapse formation, cells were soma-soma paired in $\mathrm{CM}$ containing DMSO $(0.1 \%)$. In this case normal excitatory synapses developed between RPeD1 and VD2/3 in DMSO $(100 \% ; n=5)$ (Fig. 4). It is important to note that both actinomycin D and anisomycin are active on Lymnaea neurons; however, they do not affect either action potential parameters (resting membrane potential, latency, or amplitude of action potentials) or cell viability (Feng et al., 1997). These data provide strong support for our hypothesis that CM-induced excitatory synapse formation requires both protein synthesis and transcription. 
Table 1. Excitatory synapse formation between different pairs of presynaptic and postsynaptic neurons requires CM-derived trophic molecules

Excitatory synaptic connections in vivo

\begin{tabular}{lllll}
\hline Presynaptic cell & Postsynaptic cell & Reference & DM & CM \\
\cline { 2 - 4 } RPeD1 & VD2/3 & Winlow and Benjamin, 1977 & $(-) n=24$ & $(+) n=64$ \\
VD4 & LPeD1 & Janse et al., 1985; Syed, 1988 & $(-) n=16$ & $(+) n=20$ \\
CGC & B4 & McCrohan and Benjamin, 1980 & $(-) n=8$ & $(+) n=13$ \\
RPeD11 & VD1 & Syed and Winlow, 1991 & $(-) n=7$ & $(+) n=7$ \\
VD4 & RPeD2/3 & Syed, 1988; Syed et al., 1988 & $(-) n=10$ & $(+) n=10$ \\
\hline
\end{tabular}

Identified neurons with previously demonstrated excitatory synaptic connections in vivo were soma-soma paired in vitro in either DM or CM. Evidence for the presence of $(+)$ or absence $(-)$ of excitatory synapses in vitro is indicated.

\section{CM-induced excitatory synaptogenesis between RPeD1 and VD2/3 is mediated by receptor tyrosine kinases}

To test whether CM-induced effects on excitatory synapse formation were mediated by receptor tyrosine kinases, RPeD1 and $\mathrm{VD} 2 / 3$ were soma-soma paired in CM containing receptor tyrosine kinase blockers. After 18-24 hr, only active forms of receptor tyrosine kinase inhibitors blocked CM-induced excitatory synapse formation between RPeD1 and VD2/3. Specifically, soma-soma pairing in CM containing $10 \mu \mathrm{M}$ lavendustin A (100\%; $n=10), 20 \mu \mathrm{M}$ genistein $(93 \% ; n=15), 0.1 \mu \mathrm{M} \mathrm{K} 252 \mathrm{a}$ $(93 \% ; n=12)$, or $0.1 \mu \mathrm{M} \mathrm{KT} 5926(77 \% ; n=9)$ blocked excitatory synapse formation between the soma-soma paired cells (Fig. 4). Control incubations with inactive forms of receptor tyrosine kinase blockers [10 $\mu \mathrm{M}$ lavendustin B $(100 \% ; n=13)$ and $20 \mu \mathrm{M}$ genestin $(91 \% ; n=9)$ ] had no significant effects on CM-induced excitatory synaptogenesis (Fig. 4). These data provide the first direct evidence that CM-induced excitatory synapse formation is mediated by receptor tyrosine kinases.

\section{Lymnaea EGF mimicks CM-induced effects on excitatory synaptogenesis}

The identity of molluscan trophic factors present in CM has not been fully determined. However, Lymnaea EGF has recently been purified and characterized from the albumen gland, an exocrine organ in the reproductive tract (Nagle et al., 1998). To test whether Lymnaea EGF could mimic the CM-induced effects on excitatory synapse formation between RPeD1 and VD2/3, cells were paired in DM containing various concentrations of the peptide EGF. Cell pairs maintained in DM containing $400 \mathrm{~nm}$ (Fig. 6A,B) or $800 \mathrm{~nm}$ (Fig. 6B) EGF for 18-24 hr formed excitatory synapses that were indistinguishable from those seen in vivo and in CM. Action potentials in RPeD1 induced 1:1 EPSPs in VD2/3 (Fig. 6A). These data demonstrate that Lymnaea EGF mimics $\mathrm{CM}$ in stimulating excitatory synapse formation and suggest that it (or a related peptide) plays a role in the development of excitatory synapses.

\section{EGF-induced effects on excitatory synapse formation are concentration-dependent}

To test whether EGF-induced effects on synapse formation were concentration dependent, RPeD1 and VD2/3 were soma-soma paired in DM containing different concentrations of the peptide, and synapses were tested electrophysiologically after 18-24 hr. EGF failed to promote excitatory synaptogenesis at a concentration of $100 \mathrm{nм}$ (25 nм EGF, $n=7 ; 50 \mathrm{~nm} \mathrm{EGF,} n=8 ; 100 \mathrm{nм}$ EGF, $n=11$ ) (Fig. $6 B$ ). At higher concentrations, however, EGF treatment resulted in excitatory synaptogenesis (400 nM EGF, $n=29,40 \%$; 800 nм EGF, $n=14,10 \%$ ) (Fig. $6 B$ ). The results suggest that there may be an optimal concentration of EGF for excitatory synapse formation, and that concentrations of EGF $>400$ nм may be less effective.

\section{DISCUSSION}

Together with our previously published data (Feng et al., 1997), this study demonstrated that the mechanisms underlying inhibitory and excitatory synaptogenesis between soma-soma paired Lymnaea neurons are differentially regulated by trophic factors. That is, inhibitory synapse formation appears to be regulated by cell-cell signaling mechanisms inherent to the neurons themselves, whereas excitatory synaptogenesis is contingent on extrinsic trophic factors.

The central ring ganglia-derived and heat-labile trophic molecules in CM are essential for neurite outgrowth of Helisoma (Wong et al., 1981), Aplysia (Schacher and Proshansky, 1983), and Lymnaea (Syed et al., 1990; Ridgway et al., 1991) neurons. Lymnaea is perhaps the best studied model system with regard to the identification and characterization of trophic factors in invertebrates. For example, a cysteine-rich neurotrophic factor has been purified from Lymnaea CM, which interacts with the vertebrate p75 receptor (Fainzilber et al., 1996). More recently, a mammalian homolog of the Trk receptor, termed LTrk (Lymnaea Trk), has also been identified and characterized (van Kesteren et al., 1998). Nagle and co-workers (1998) susequently isolated EGF from Lymnaea albumen glands, which has also been shown to promote neurite outgrowth from Lymnaea neurons (Hermann et al., 1998). The Lymnaea EGF gene is expressed at its highest levels during CNS development in juvenile Lymnaea, but expression is virtually undetectable in the adult CNS; moreover, EGF gene expression is up-regulated after nerve injury (G. T. Nagle, personal communication).

These data support the hypothesis that EGF may play a role in vivo in neurite outgrowth and excitatory synapse formation during early development of the CNS and during nerve injury repair processes. In addition to the above studies demonstrating the presence and function of native trophic factors and receptors in Lymnaea, various mammalian neurotrophic factors have also been shown to affect neurite outgrowth and synapse formation between Lymnaea neurons. For example, nerve growth factor (NGF; Ridgway et al., 1991) and ciliary neurotrophic factor (CNTF; Syed et al., 1996) promoted neurite outgrowth from Lymnaea neurons. However, NGF- but not CNTF-induced neurite outgrowth resulted in synapse formation between identified Lymnaea neurons (Syed et al., 1996), suggesting that these two developmental programs may be differentially regulated by tro- 


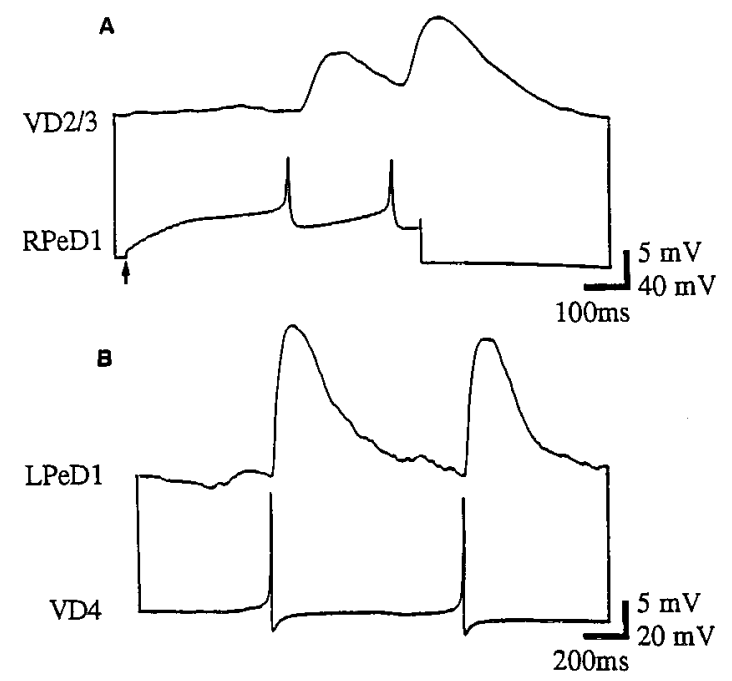

Figure 3. Appropriate excitatory synapses are reestablished between soma-soma paired neurons in CM. After $18-24 \mathrm{hr}$ of soma-soma pairing in $\mathrm{CM}$, simultaneous intracellular recordings showed that action potentials in RPeD1 produced 1:1 EPSPs in VD2/3 $(A)$. $B$, Similarly, action potentials in VD4 produced 1:1 EPSPs in LPeD1.

phic molecules. Taken together, the above studies demonstrate that mammalian homologs of a variety of vertebrate trophic factors and their receptors are not only conserved in Lymnaea but are also functional. Nevertheless, the cellular and molecular mechanisms by which endogenous trophic factors and receptors and their mammalian counterparts promote neurite outgrowth and synapse formation remain relatively unexplored.

In the present study, we have demonstrated that excitatory synapse formation between different presynaptic and postsynaptic partners requires $\mathrm{CM}$, suggesting that trophic support is fundamental to all excitatory synapses studied in this model to date. Because the CM-induced effects on excitatory synapse formation in Lymnaea were only partially (40\%) mimicked by EGF, other as yet unidentified trophic molecules present in $\mathrm{CM}$ are presumably involved. Nevertheless, this study is the first to extend the role of EGF from cell proliferation, survival, and neurite outgrowth (Yamada et al., 1997) to synaptogenesis.

Neurotrophic factors affect neuronal survival and promote neurite outgrowth via a family of receptor tyrosine kinases (Schlessinger and Ullrich, 1992). Consistent with these studies, our data demonstrated that CM-induced excitatory synapse formation was blocked by active (lavendustin A) but not inactive (lavendustin B) forms of a receptor tyrosine kinase inhibitor (Onoda et al., 1989). Similarly, we showed that K252a (and its derivative K T5926), which blocks a variety of vertebrate tyrosine kinases (Oberstar et al., 1997), also suppressed CM-induced excitatory synapse formation. Moreover, CM-induced excitatory synapse formation was blocked by the nonspecific receptor tyrosine kinase inhibitor Genistein at a lower concentration (20 $\mu \mathrm{M})$ than has been shown to block synapse formation in vertebrates (100 $\mu \mathrm{M}$; Simpson and Morris, 1995). The inactive structural analog of genestein, genistin $(20 \mu \mathrm{M})$, did not block CMinduced excitatory synapse formation. Interestingly, lavendustin A failed to block inhibitory synapse formation between VD4 and RPeD1 (P. Lovell, unpublished observations), suggesting that the receptor tyrosine kinase-mediated synaptogenesis may be specific to excitatory synapses. Although the specificity of the above compounds for various subtypes of Lymnaea kinases has not yet

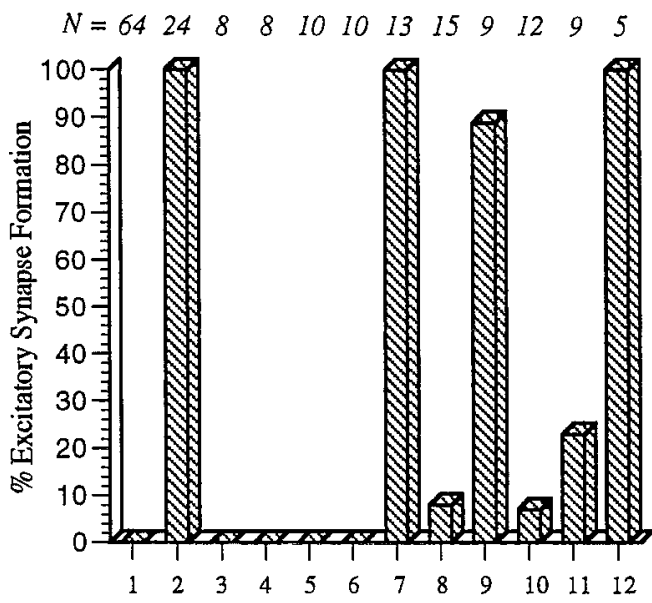
1. $\mathrm{DM}$
7. $\mathrm{CM}+$ Lavendustin $\mathrm{B}$
2. $\mathrm{CM}$
8. $\mathrm{CM}+$ Genistein
3. $\mathrm{CM}+$ Anisomycin
9. $\mathrm{CM}+$ Genistin
4. $\mathrm{CM}+$ Actinomycin D
10. $\mathrm{CM}+\mathrm{K} 252 \mathrm{a}$
5. CM (Heat Inactivated)
11. $\mathrm{CM}+\mathrm{KT} 5926$
6. $\mathrm{CM}+$ Lavendustin $\mathrm{A}$
12. $\mathrm{CM}+\mathrm{DMSO}$

Figure 4. CM-induced synaptogenesis between RPeD1 and VD2/3 requires transcription, de novo protein synthesis, and receptor tyrosine kinases. In DM, an appropriate excitatory synapse fails to develop between RPeD1 and VD2/3. When paired in CM, however, appropriate excitatory synapses reestablish between soma-soma paired RPeD1 and $\mathrm{VD} 2 / 3$. CM-induced excitatory synapse formation is blocked, however, when paired cells are incubated in CM containing either anisomycin (protein synthesis inhibitor) or actinomycin D (protein transcription inhibitor). Heat-inactivated $\mathrm{CM}$ (boiled at $100^{\circ} \mathrm{C}$ for $20 \mathrm{~min}$ ) also fails to support excitatory synapse formation. CM-induced excitatory synapse formation was partially or completely blocked by the addition of receptor tyrosine kinase inhibitors (lavendustin A, genistein, K252a, or KT5926) but not by inactive forms of receptor tyrosine kinase inhibitors (lavendustin B and genistin). Similarly, addition of the carrier solution (DMSO) to $\mathrm{CM}$ did not perturb synapse formation between RPeD1 and VD2/3. $N$ values for each experiment are indicated above the data bars.

been resolved, our data nevertheless suggest that CM-induced excitatory synapse formation is indeed mediated by receptor tyrosine kinases.

In the peripheral nervous system, retrograde instructive signals from target tissues can regulate neurotransmitter phenotype by altering the expression of enzymes involved in neurotransmitter and neuropeptide synthesis (Landis, 1990). More recently, it has been suggested that neurotransmitter phenotype selection in the CNS may also be regulated by neurotrophic factors (for review, see Zhou and Bradford, 1997). Based on these elegant studies, one could argue that the differential regulation of excitatory and inhibitory synapse formation between RPeD1 and VD2/3 results from trophic factor-induced alteration in transmitter phenotypic characteristics of the presynaptic neuron. However, this is not supported by our data for the following reason. The presynaptic neuron (RPeD1), which was the main focus of our experiments, is known to contain and release dopamine as its sole neurotransmitter. Moreover, when paired with its inhibitory partner (VD4), RPeD1 is capable of establishing its inhibitory synapse in both DM and CM (Feng et al., 1997). Because inhibitory synaptic transmission between RPeD1 and VD4 is mediated by dopamine, these results suggest that the dopaminergic phenotype is expressed under both experimental conditions (CM and DM). Therefore, these data demonstrate that the presynaptic, dopami- 

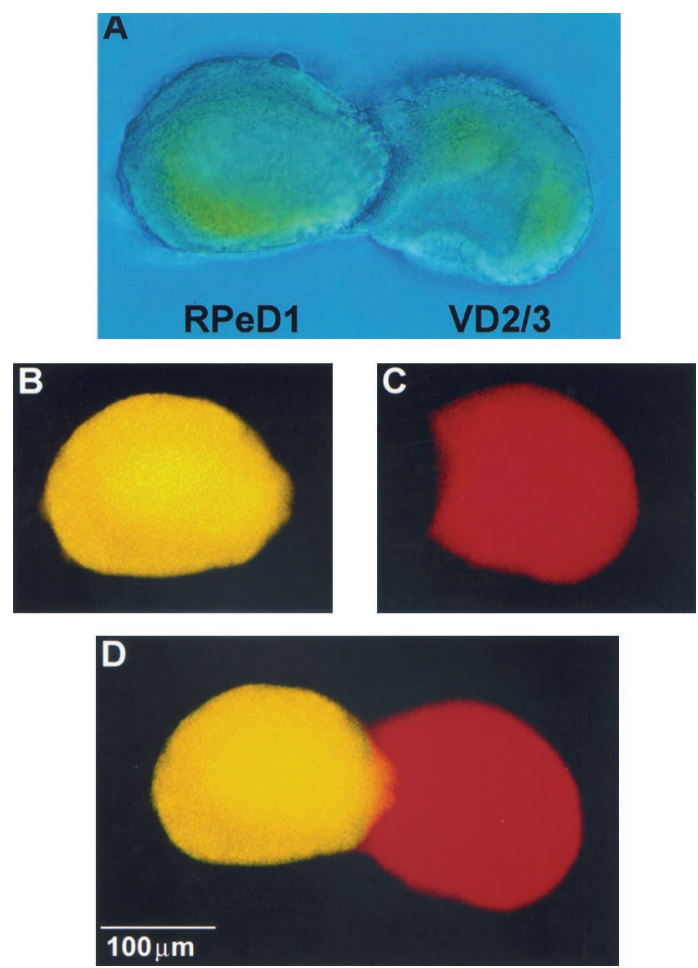

Figure 5. CM-induced excitatory synaptogenesis does not involve neurite outgrowth from soma-soma paired cells. To test whether CMinduced excitatory synapse formation between soma-soma paired cells $(A)$ involved neurite outgrowth, both presynaptic (RPeD1) and postsynaptic (VD2/3) neurons were injected with fluorescent markers. Specifically, neurons were injected iontophoretically with either Lucifer yellow (RPeD1; $B)$ or sulforhodamine $(\mathrm{VD} 2 / 3 ; C)$ and viewed by fluorescence microscopy. Neither RPeD1 $(B, D)$ nor $\mathrm{VD} 2 / 3(C, D)$ exhibited neurite outgrowth in CM.

nergic secretory machinery is fully functional in the absence of trophic factors. Similarly, postsynaptic VD2/3 neurons maintained in either CM or DM exhibit an electrophysiological response to exogenous dopamine (data not shown), suggesting that the expression of postsynaptic dopamine receptors in vitro does not depend on trophic factors. These findings are consistent with earlier studies on Aplysia neurons in which an inappropriate inhibitory synapse developed between identified neurons R2 and $\mathrm{R} 15$ in cell culture (in vivo, the cholinergic synaptic transmission between these neurons is excitatory) (Schacher et al., 1985). Because exogenously applied acetylcholine induced an excitatory response in an isolated neuron, $\mathrm{R} 15$, these data show that despite the presence of appropriate cholinergic (excitatory) receptors, R2 makes an inappropriate inhibitory synapse with R15.

Neurotrophic factors can regulate dendritic growth in the developing visual cortex (McAllister et al., 1995, 1996) and are involved in the remodeling of axonal branching patterns in vitro (Cohen-Corey and Fraser, 1995). The fluorescence microscopic data presented in this study strongly suggest that the CM- and EGF-induced excitatory synapse formation does not involve neuronal spouting. However, detailed ultrastructural analysis is required to definitively rule out this possibility.

Recent evidence suggests that neurotrophic factors modulate the electrical activity patterns of a variety of neurons (Kim et al., 1994; McAllister et al., 1996). Consistent with this role are their effects on a variety of ion channels that subsequently regulate the intrinsic membrane properties (Berninger and Poo, 1996). Con-
A. DM (EGF 400nM)
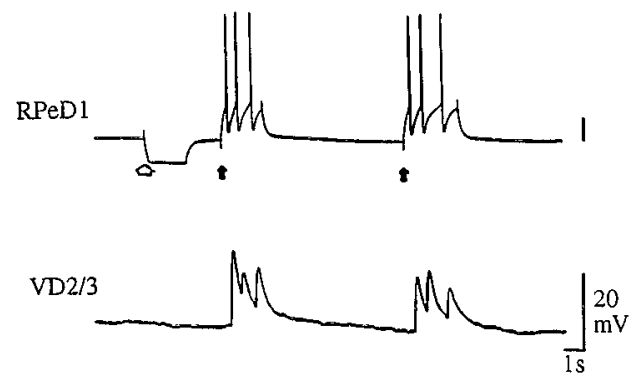

B. EGF

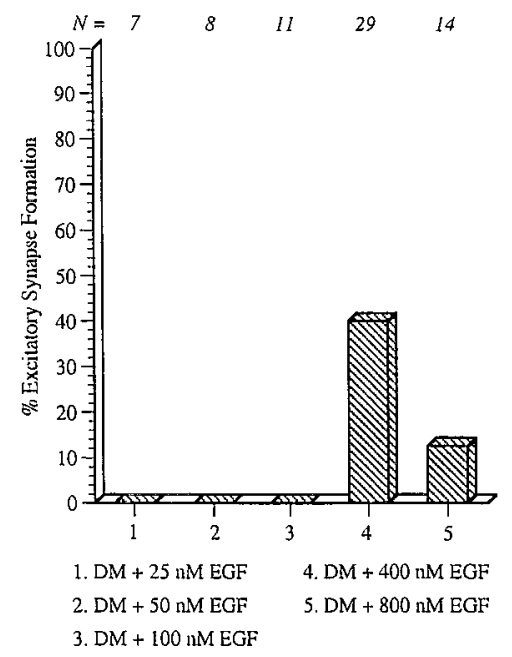

Figure 6. CM-induced effects on excitatory synaptogenesis are mimicked by Lymnaea EGF. Identified neurons RPeD1 and VD2/3 were somasoma paired in DM containing various concentrations of Lymnaea EGF. $A$, EGF (400 nM) induced synaptogenesis between soma-soma paired $\mathrm{RPeD} 1$ and VD2/3. Specifically, action potentials in RPeD1 (solid arrow) produced 1:1 EPSPs in VD2/3. The injection of a hyperpolarizing current in RPeD1 (open arrow) did not reveal electrical coupling between the cells. $B$, The relationship between EGF concentration and excitatory synaptogenesis between RPeD1 and VD2/3 was most similar to an inverted U-shaped function.

versely, neuronal activity patterns can also regulate the expression and release of various neurotrophic factors (Funakoshi et al., 1995). Finally, Boulanger and Poo (1999) have recently shown that neurotrophin-induced facilitation of the neuromuscular synapse is contingent on presynaptic activity. Our preliminary results have shown that acute $\mathrm{CM}$ treatment increases the spontaneous electrical activity patterns of soma-soma paired Lymnaea neurons (Bjorgum et al., 1998). Based on these and previously published studies, our working hypothesis is that CM- and EGFinduced excitatory synapse formation may involve changes in the activity patterns of both soma-soma paired cells. Because activity patterns are more likely to influence excitatory than inhibitory synapses, it therefore seems plausible that the trophic factorinduced synaptogenesis may involve activity-dependent mechanisms.

\section{REFERENCES}

Berninger B, Poo M-M (1996) Fast actions of neurotrophic factors. Curr Opin Neurobiol 6:324-330.

Bjorgum MC, Hamakawa T, Takasaki M, Syed NI (1998) Brainconditioned medium induced excitatory synaptogenesis between identified Lymnaea neurons may require activity dependent mechanisms. Soc Neurosci Abstr 24:791. 
Boulanger L, Poo M-M (1999) Presynaptic depolarization facilitates neurotrophin-induced synaptic potentiation. Nat Neurosci 2:345-351.

Cohen-Corey S, Fraser SE (1995) Effects of brain-derived neurotrophic factor on optic axon branching and remodeling in vivo. Nature 378:192-196.

Fainzilber M, Smit AB, Syed NI, Wildering WC, Hermann PM, van der Schors RC, Jiménez C, Li KW, van Minnen J, Bulloch AGM, Ibáñez CF, Geraerts WPM (1996) CRNF, a molluscan neurotrophic factor that interacts with the p75 neurotrophin receptor. Science 274:1540-1543.

Feng Z-P, Klumperman J, Lukowiak K, Syed NI (1997) In vitro synaptogenesis between the somata of identified Lymnaea neurons requires protein synthesis but not extrinsic growth factors or sustrate adhesion molecules. J Neurosci 17:7839-7849.

Funakoshi H, Bellurado N, Arenas E, Yamamoto Y, Casabona A, Persson H, Ibanez CF (1995) Muscle-derived neurotrophin-4 as an activity-dependent trophic signal for adult motor neurons. Science 268:1459-1499.

Hermann PM, Wildering WC, Painter SD, Nagle GT, Bulloch AGM (1998) Molluscan epidermal growth factor: neurotrophic actions. Soc Neurosci Abstr 24:106.

Janse C, van der Wilt CJ, van der Plas J, van der Roest M (1985) Central and peripheral neurons involved in oxygen perception in the pulmonate snail Lymnaea stagnalis (Mollusca Gastropoda). Comp Biochem Physiol 82A:459-467.

Kang H, Schuman EM (1995) Long-lasting neurotrophin-induced enhancement of synaptic transmission in the adult hippocampus. Science 267:1658-1662.

Kim HG, Wang T, Olafsson P, Lu B (1994) Neurotrophin-3 potentiates neuronal activity and inhibits G-aminobutyratergic synaptic transmission in cortical neurons. Proc Natl Acad Sci USA 91:12341-12345.

Landis SC (1990) Target regulation of neurotransmitter phenotype. Trends Neurosci 13:344-350.

Leßmann V, Gottmann K, Heumann R (1994) BDNF and NT-4/5 enhance glutamatergic synaptic transmission in cultured hippocampal neurones. NeuroReport 6:21-25.

Levine ES, Dreyfus CF, Black IB, Plummer MR (1995) Brain-derived neurotrophic factor rapidly enhances synaptic transmission in hippocampal neurons via postsynaptic tyrosine kinase receptors. Proc Natl Acad Sci USA 92:8074-8077.

Li YX, Zhang Y, Lester HA, Schuman EM, Davidson N (1998) Enhancement of neurotransmitter release induced by brain-derived neurotrophic factor in cultured hippocampal neurons. J Neurosci 18:10231-10240.

Liou JC, Yang RS, Fu WM (1997) Regulation of quantal secretion by neurotrophic factors at developing motoneurons in Xenopus cell cultures. J Physiol (Lond) 503:129-139.

Lohof AM, Ip NY, Poo M-M (1993) Potentiation of developing neuromuscular synapses by the neurotrophins NT-3 and BDNF. Nature 363:350-353.

Magoski NS, Bulloch AGM (1997) Localization, physiology, and modulation of a molluskan dopaminergic synapse. J Neurobiol 33:247-264.

Magoski NS, Bauce LG, Syed NI, Bulloch AGM (1995) Dopaminergic transmission between identified neurons from the mollusc Lymnaea stagnalis. J Neurophysiol 74:1287-1300.

McAllister AK, Lo DC, Katz LC (1995) Neurotrophins regulate dendritic growth in developing visual cortex. Neuron 15:323-331.

McAllister AK, Katz LC, Lo DC (1996) Neurotrophin regulation of cortical dendritic growth requires activity. Neuron 18:767-778.

McCaman MW, Ono JK, McCaman RE (1979) Dopamine measurements in molluscan ganglia and neurons using a new, sensitive assay. $\mathbf{J}$ Neurochem 32:1111-1113.

McCrohan CR, Benjamin PR (1980) Synaptic relationships of the cerebral giant cells with motorneurones in the feeding system of Lymnaea stagnalis. J Exp Biol 85:169-186.

Nagle GT, Akalal D-BG, ter Maat A, de With ND, Painter SD (1998) Molluscan epidermal growth factor: purification and characterization of a neurotrophic factor in the pulmonate Lymnaea. Soc Neurosci Abstr 24:106.

Oberstar JV, Challacombe JF, Roche FK, Letourneau PC (1997) Concentration-dependent stimulation and inhibition of growth cone behavior and neurite elongation by protein kinase inhibitors. J Neurobiol 33:161-171.

Onoda T, Linuma H, Sasaki Y, Hamada M, Isshiki K, Naganawa H,
Takeuchi T, Tatsuta K, Umezawa K (1989) Isolation of a novel tyrosine kinase inhibitor, lavendustin A, from Streptomyces griseolavendus. J Nat Prod 52:1252-1257.

Oppenheim RW (1991) Cell death during development of the nervous system. Annu Rev Neurosci 14:453-501.

Purves D, Lichtman JW (1985) Principles of neural development. Sunderland, MA: Sinauer.

Ridgway RL, Syed NI, Lukowiak K, Bulloch AGM (1991) Nerve growth factor (NGF) induces sprouting of specific neurons of the snail, Lymnaea stagnalis. J Neurobiol 22:377-390.

Schacher S, Proshansky E (1983) Neurite regeneration by Aplysia neurons in dissociated cell culture: modulation by Aplysia hemolymph and the presence of the initial axonal segment. J Neurosci 3:2403-2413.

Schacher S, Rayport G, Ambron RT (1985) Giant Aplysia neuron R2 reliable forms strong chemical connections in vitro. J Neurosci 5:2851-2865.

Schlessinger J, Ullrich A (1992) Growth factor signaling by receptor tyrosine kinases. Neuron 9:383-391.

Schuman EM (1997) Synapse specificity and long-term information storage. Neuron 18:339-342.

Simpson CS, Morris BJ (1995) Stimulation of zif/268 gene expression by basic fibroblast growth factor in primary rat striatal cultures. Neuropharmacology 34:515-520.

Stoop R, Poo M-M (1995) Potentiation of transmitter release by ciliary neurotrophic factors requires somatic signaling. Science 267:695-699.

Stoop R, Poo M-M (1996) Synaptic modulation by neurotrophic factors: differential and synergistic effects of brain-derived neurotrophic factor and ciliary neurotrophic factor. J Neurosci 16:3256-3264.

Syed NI (1988) Neural control of locomotion in Lymnaea. PhD Thesis, University of Leeds.

Syed NI, Winlow W (1989) Morphology and electrophysiology of neurons innervating ciliated locomotor epithelium in Lymnaea stagnalis (L). Comp Biochem Physiol 93A:633-644.

Syed NI, Winlow W (1991) Respiratory behaviour in the pond snail Lymnaea stagnalis. II. Neural elements of the central pattern generator (CPG). J Comp Physiol [A] 169:557-568.

Syed NI, Harrison D, Winlow W (1988) Locomotion in Lymnaea-role of serotonergic motorneurons controlling the pedal cilia. Symp Biol Hung 36:387-402.

Syed NI, Bulloch AGM, Lukowiak K (1990) In vitro reconstruction of the respiratory central pattern generator of the mollusk Lymnaea. Science 250:282-285.

Syed N, Richardson P, Bulloch AGM (1996) Ciliary neurotrophic factor unlike nerve growth factor, supports neurite outgrowth but not synapse formation by adult Lymnaea neurons. J Neurobiol 29:293-303.

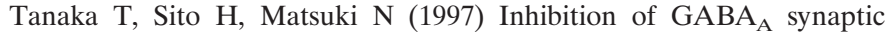
responses by brain-derived neurotrophic factor (BDNF) in rat hippocampus. J Neurosci 17:2959-2966.

Thoenen H (1991) The changing scene of neurotrophic factors. Trends Neurosci 14:165-170.

Thoenen H (1995) Neurotrophins and neuronal plasticity. Science 270:593-598.

van Kesteren RE, Fainzilber M, Hauser G, van Minnen J, Vreugdenhil E, Smit AB, Ibánez CF, Geraerts WPM, Bulloch AGM (1998) Early evolutionary origin of the neurotrophin receptor family. EMBO J 17:2534-2542.

Vicario-Abéjon C, Collin C, McKay RDG, Segal M (1998) Neurotrophins induce formation of functional excitatory and inhibitory synapses between cultured hippocampal neurons. J Neurosci 18:7256-7271.

Wang T, Xie K, Lu B (1995) Neurotrophins promote maturation of developing neuromuscular synapses. J Neurosci 15:4796-4805.

Wang XH, Poo M-M (1997) Potentiation of developing synapses by postsynaptic release of neurotrophin-4. Neuron 19:825-835.

Winlow W, Benjamin PR (1977) Postsynaptic effects of a multiaction giant interneurone on identified snail neurones. Nature 268:263-265.

Wong RG, Hadley RD, Kater SB, Hauser GC (1981) Neurite outgrowth in molluscan organ and cell cultures: the role of conditioning factor(s). J. Neurosci 1:1008-1021.

Yamada M, Ikeuchi T, Hatanaka H (1997) The neurotrophic action and signalling of epidermal growth factor. Prog Neurobiol 51:19-37.

Zhou J, Bradford HF (1997) Nerve growth factors and the control of neurotransmitter phenotype selection in the mammalian central nervous system. Prog Neurobiol 53:27-43. 